\title{
Transmesenteric robot-assisted pyeloplasty for ureteropelvic junction obstruction in horseshoe kidney
}

\author{
Aaron M. Potretzke ${ }^{1}$, Anand Mohapatra ${ }^{1}$, Jeffrey A. Larson ${ }^{1}$, Brian M. Benway ${ }^{2}$ \\ ${ }^{1}$ Washington University School of Medicine, Division of Urologic Surgery, St. Louis, MO, USA; ${ }^{2}$ Urology \\ Academic Practice, Cedars-Sinai Medical Center, Los Angeles, CA, USA
}

\section{INTRODUCTION}

Transmesenteric laparoscopic pyeloplasty has previously been reported in two pediatric patients. To our knowledge, there has yet to be a report of a robotic-assisted transmesenteric pyeloplasty. We sought to present a video display and step-by-step demonstration of this approach.

\section{CASE}

The patient is a 28-year old female with an obstructed ureteropelvic junction (UPJ) of the left moiety of a horseshoe kidney. The Da Vinci S robotic platform was used. After transperitoneal access was obtained, a window in the mesentery was identified, and the renal pelvis was exposed. A dismembered, spatulated pyeloplasty was performed with transposition of the UPJ to a more dependent portion of the renal pelvis. A ureteral stent and closed surgical drain were placed.

\section{RESULTS}

The case was completed without complications. Operative time was 207 minutes. Foley catheter and drain were removed on postoperative days 1 and 2, respectively. The patient was discharged home on postoperative day 2 , and the ureteral stent was removed after 8 weeks. Her diuretic T1/2 improved from 39 to 16 minutes. The differential function of the left moiety improved from $31 \%$ to $42 \%$.

\section{CONCLUSIONS}

Robot-assisted transmesenteric pyeloplasty is safe and feasible for the management of UPJ obstruction in select patients with a horseshoe kidney. Future study of its use in a larger number of patients is necessary to define its role in this unique population.

\section{CONFLICT OF INTEREST}

None declared.

\section{ARTICLE INFO}


Submitted for publication:

June 06, 2015

Accepted after revision:

July 17, 2015
Correspondence address:

Aaron M. Potretzke, MD

Division of Urology, Washington University School of Medicine 4960 Children's Place

St. Louis, M0, 63110, USA

Fax: +1 314 367-5016

E-mail: potretzkea@wudosis.wustl.edu

\section{EDITORIAL COMMENT}

This is a well-done video that adds to the literature in the fledgling field of minimally-invasive reconstructive urology. This video should encourage urologists to counsel their patients that this procedure can be performed safely using minimally-invasive techniques with good outcomes. The difficulty with laparoscopic and/or robotic ap- proaches to upper ureteric strictures is when direct visualization shows that the fibrosis extends beyond what was thought preoperatively. In this situation, the surgeon is faced with the choice of trying to reposition the patient for minimally-invasive psoas hitch/Boari flap, opening, or trying to obtain more length of ureter or bridge a gap that may cause undue tension. While the outcome here is excellent, proper patient selection is key.

Lucas Wiegand, MD Assistant Professor University of South Florida Morsani College of Medicine Tampa, FL, USA E-mail:lwiegand@health.usf.edu 